\title{
471 PHARMACOKINETICS OF FIRST AND REPEATED DOSING OF NON-IRAE-INDUCING ANTI-CTLA-4 MONOCLONAL ANTIBODY ONC-392 IN ADVANCED CANCER PATIENTS
}

${ }^{1}$ Hung-Yen Chou*, ${ }^{2}$ Tianhong Li, ${ }^{2}$ Karen Kelly, ${ }^{2}$ Anthony Martinez, ${ }^{2}$ Stacy Joo, ${ }^{3}$ Mei Tang, ${ }^{1}$ Martin Devenport, ${ }^{1}$ Yang Liu, ${ }^{1}$ Pan Zheng. ${ }^{1}$ OncoC4, Inc., Rockville, MD, USA; ${ }^{2}$ UC Davis Comprehensive Cancer Center, Sacramento, CA, USA; ${ }^{3}$ Greater Baltimore Medical Center, Baltimore, MD, USA

Background ONC-392 preserves CTLA-4 recycling and thereby maintains its physiological immune tolerance checkpoint function while allowing more efficient and selective elimination of tumor-infiltrating regulatory $\mathrm{T}$ cells. The safety data in the first-in-human trial showed that ONC-392 is safe and well tolerated with no observed immunotherapy-related adverse events (irAE). Serum samples were used to determine pharmacokinetic parameters of ONC-392 to establish systemic drug exposure.

Methods Samples from the first and third dosing cycles were collected at predose and $0.5,6,24,48,192,360$, and 504 hours postdose. For other dosing cycles, predose and 0.5 hour postdose samples were collected. Serum ONC-392 concentrations were measured by ELISA and the PK parameters were analyzed under noncompartmental condition using linear trapezoidal method.

Results Systemic exposure of ONC-392 is positively correlated to dosing concentration and number of doses. Mean Cmax and AUC $0-504 \mathrm{hr}$ values increase proportionately to dosing concentrations from $0.1 \mathrm{mg} / \mathrm{kg}$ to $10 \mathrm{mg} / \mathrm{kg}$. Dose ratio in cycle 1 is 1:3:30:100. The mean cycle $1 \mathrm{Cmax}$ and AUC $0-504 \mathrm{hr}$ ratios are 1:3.34:31.32:106.28 and 1:3.13:28.46:100.63 respectively. The Cmax in patients receiving one or more doses of ONC-392 at $3 \mathrm{mg} / \mathrm{kg}$ is $89 \pm 16 \mu \mathrm{g} / \mathrm{mL}$. The Cmax in patients receiving one or more doses of ONC-392 at $10 \mathrm{mg} / \mathrm{kg}$ is $259 \pm 55 \mu \mathrm{g} / \mathrm{mL}$. Inclusive of all dosing concentrations $(0.1$, $0.3,1,3,10 \mathrm{mg} / \mathrm{kg}$ ) and cycles, Tmax is between $1.5-6$ hours with one outlier observed at 24 -hour postdose. The $t^{1 / 2}$ range from 201 to 478 hours ( 8 - 20 days). The cycle 1 mean of $\mathrm{t}^{1 / 2}$ for $0.1,0.3,3,10 \mathrm{mg} / \mathrm{kg}$ dosing concentrations are 411.02 , $359.25,246.22,355.01$ hours respectively. A direct comparison between first and third cycle in the $3 \mathrm{mg} / \mathrm{kg}$ dosing group confirms ONC-392 accumulation in repeated dosing. The trough levels (Cmin) in patients receiving one or multiple doses of ONC-392 at $3 \mathrm{mg} / \mathrm{kg}$ and $10 \mathrm{mg} / \mathrm{kg}$ are between $12-$ $51 \mu \mathrm{g} / \mathrm{mL}$ and $49-71 \mu \mathrm{g} / \mathrm{mL}$ respectively. Lastly, inclusive of all dosing concentrations $(0.1,0.3,1,3,10 \mathrm{mg} / \mathrm{kg})$ and cycles, MRT range from 307.91-655.04 hours, $\mathrm{Vz}$ range from $0.0305-0.0726 \mathrm{mg} /(\mu \mathrm{g} / \mathrm{mL})$, and $\mathrm{Cl}$ range from $0.000052-$ $0.00019 \mathrm{mg} /(\mu \mathrm{g} / \mathrm{mL}) / \mathrm{h}$.

Conclusions Intravenous infusion of ONC-392 provide adequate and dose-dependent exposure over extended period. Overall exposure is comparable or higher than those reported by others using different anti-CTLA- 4 antibodies. The apparent lack of irAE in ONC-392 recipients despite the high exposure indicates intrinsic safety and tolerability of ONC392.

http://dx.doi.org/10.1136/jitc-2021-SITC2021.471 